\title{
Study of the conrod deformation during piston interaction with liquid in the internal combustion engine cylinder
}

\author{
Sergey A. Dmitriev and Alexander E. Khrulev* \\ Aerospace Institute, National Aviation University, Kyiv 03058, Ukraine. \\ Phone: +380961632183
}

ABSTRACT - The paper analyzes the deformation of the connecting rod stem with buckling due to water ingress into the internal combustion engine cylinder (the so-called hydrolock). A method is presented that has been developed to perform calculations of stem deformation in the process of compressing air with liquid in an internal combustion engine cylinder. The method is based on solving a system of differential equations for pressure and temperature in the cylinder, followed by calculating the compression force acting on the connecting rod. A carried-out simulation of the compression process demonstrates the dependence of the air pressure in the cylinder, the stress and the strain of the connecting rod on the fill ratio of the combustion chamber with liquid. The calculations performed according to the classical theory of resistance of materials have shown that the connecting rod with the buckling of the stem begins to deform when the liquid fills the combustion chamber to a minimum of $80 \%$. With the increase in the amount of liquid, the deformation of the conrod increases, and when the level of liquid filling is so significant that it exceeds the volume of the combustion chamber, the conrod stem deformation reaches extreme values. It is shown that under these conditions after the hydrolock occurs the engine may fail due to the piston wedging the crankshaft in the bottom dead center position.
ARTICLE HISTORY

Revised: $1^{\text {st }}$ Dec 2019

Accepted: $6^{\text {th }}$ Dec 2019

\section{KEYWORDS}

Internal combustion

engine;

hydrolock;

conrod;

failure;

damage;

buckling.

\section{INTRODUCTION}

The study of damage and failure of internal combustion engines shows that one of the common causes of the most severe damage that causes engine failure in operation (up to it becoming non-repairable) is liquid entering the cylinder [1]. This phenomenon is generally called hydraulic lock [2] or, more correctly, hydrostatic lock (hydrolock).

Practice also shows that, under certain conditions, various liquids can flux into the cylinder of the internal combustion engine [3]. Thus, the most common operational cause of the "water" hydrolock in the cylinder is the flux of water from the environment through the intake or the exhaust systems. Turbocharged engines encounter an "oil" hydrolock when the engine oil that has accumulated in the aggregates or nodes of the intake system enters the cylinder. Less common is the "fuel" hydrolock which happens as a result of a malfunction of the fuel system components, while the rarest is the hydrolock caused by the coolant entering the cylinder.

Since at least one of the mentioned types of hydraulic locks is possible in transport ICEs of any type, purpose and scope, studies of the symptoms, causes and cause-effect relationships of this phenomenon are quite relevant not only in the field of the vehicle operation or repair, but also in their construction.

Despite having completely different causes, all these types of hydrolock demonstrate a similar damage process. For example, it is known that hydrolock occurs when the combustion chamber is filled with liquid. The piston then will, most likely, not be able to reach the extreme upper position (top dead center) - it will thrust against the liquid.

However, despite hydrolock being frequently referenced in numerous sources, the provided quantitative estimates or characteristics of this phenomenon in most cases are incomplete. The description of the hydrolock is often limited to a brief mention of some of its symptoms only, which cannot be considered sufficient to practically determine all signs of damage and identify the cause of failure in any given engine. Besides, the known sources [4, 5] provide no data on the effect the amount of liquid has on the value and nature of the parts deformation - most importantly, the connecting rods. Moreover, the known studies of the connecting rod deformation with the stem buckling [6, 7] mainly concern only with the deformation itself and do not review in detail the process that causes it. As a result, the investigation of possible reasons for damage caused by hydrolock - even after the final state of the parts has been analyzed - is hindered by the difficulty in determining the amount of liquid in the cylinder as well as associating the determined amount with the existing symptoms of a specific cause of a hydrolock, which is required to subsequently identify the cause-and-effect relationships of the damage with a manufacturing defect or operational damage.

Meanwhile, the practice of investigating the causes of malfunctions of internal combustion engines [1, 3] shows that in some cases, when the cause of the damage as well as the type of hydrolock (water, oil or fuel) are not obvious, any additional data and symptoms cannot be superfluous for the researcher. 
In accordance with this, the objective of this work is to study the phenomenon of hydrolock as a process in the cylinder of internal combustion engine in order to identify the quantitative characteristics of this phenomenon. The contribution of the study lies in obtaining quantitative data on the connection between the conrod buckling and the liquid filling of the combustion chamber, which was done for the first time.

\section{METHODS AND MATERIALS}

In order to derive score equations for the phenomenon under study, it is necessary to examine some of its features. The experience of analyzing failures in real engines [8] shows that when liquid enters the cylinder, the connecting rod becomes the weakest link of the conrod-piston group and experiences significant axial compressive loads, as a result of which the stem of the connecting rod may become unstable and deform [9].

\section{Features of Hydrolock Damage to Parts}

An analysis of cases known from the practice of investigating the causes of engine malfunction indicates that the deformation of conrod may vary in size, with the symptoms of buckling mainly occurring in the direction of the axis of the crankshaft rotation, because in this direction the stem rigidity is usually smaller than in the rotate direction (Figure1) due to the fact that the width of the stem profile is smaller than its height. However, although less common, the stem profile of small width is also vulnerable to buckling which might occur in the plane of rotation.

Obviously, the buckling should be accompanied not only by the curvature of the conrod stem, but also by its axial compression. As a result, the center distance (the distance between the axles of the crank and piston heads of conrod) decreases.

In order to obtain full data on the phenomenon, it is necessary to carry out accurate measurements of the deformed connecting rod. However, this task is very difficult to accomplish, and in most practical cases not possible at all, since the subsequent fatigue failure of the stem from abnormal bending loads (in fact, this is what operation of the engine with a bent connecting rod usually leads to) will leave you with only the fragments of the conrod.

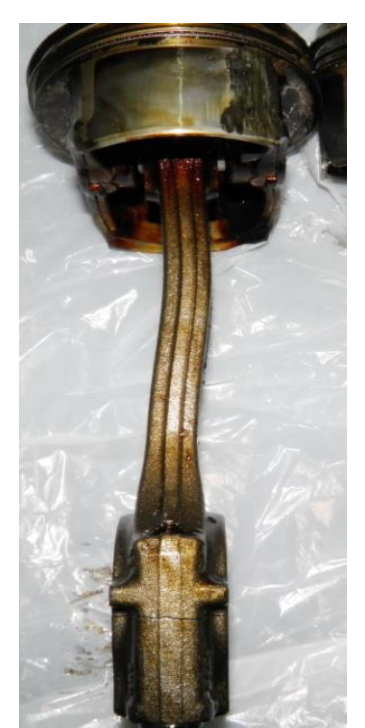

(a)

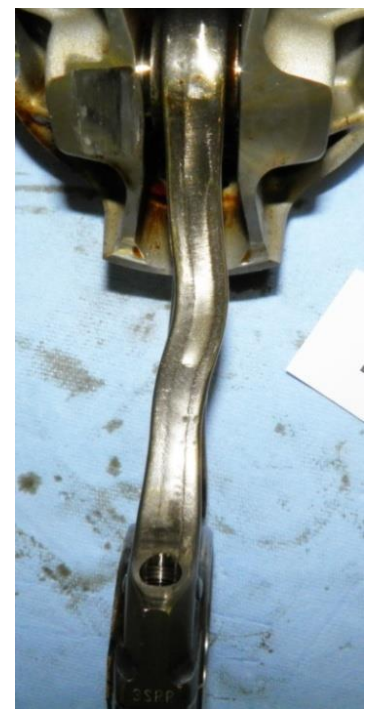

(b)

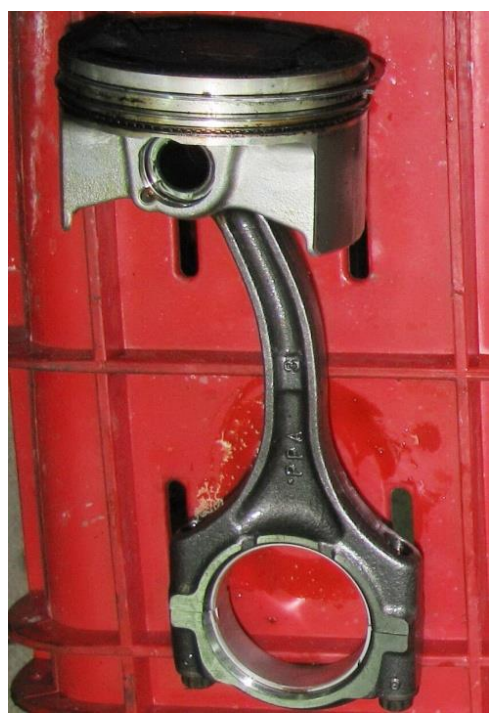

(c)

Figure 1. The buckling of the conrod stem in the direction of the axis of the crankshaft caused by a hydrolock in the internal combustion engine cylinder.

The buckling of the conrod stem in the direction of the axis of the crankshaft caused by a hydrolock in the internal combustion engine cylinder may be relatively weak (a) or strong (b). Buckling of the conrod stem in the plane of the crank rotation (c) is possible only with a small height of the stem profile i.e. small ratio of height (c) to width of the profile (a) and (b).The data obtained is a result of analysis of a great number of engines [8].

However, measuring the wrecks, like the connecting rod itself, is not required. In order to determine the value of the stem deformation along its length, the indirect method [9] is quite sufficient, which lies in measuring the height of the carbon footprint on the upper part of the cylinder and comparing it to those cylinders where no hydrolock has been detected (Figure 2), that allows you to definitively measure the conrod stem deformation: a) by $1.5 \mathrm{~mm}$ (the images of two adjacent cylinders were combined), b) $2.5 \mathrm{~mm}$ and c) $3.5 \mathrm{~mm}$.

It is clear that the connecting rod deformation may be significantly greater than that indicated in Figure 2; in that case there is a danger of the crankshaft wedging due to the piston landing onto the crankshaft counterweights near the bottom dead center (Figure 3 ) or even the deformed conrod stem wedging onto the bottom cylinder edge. 


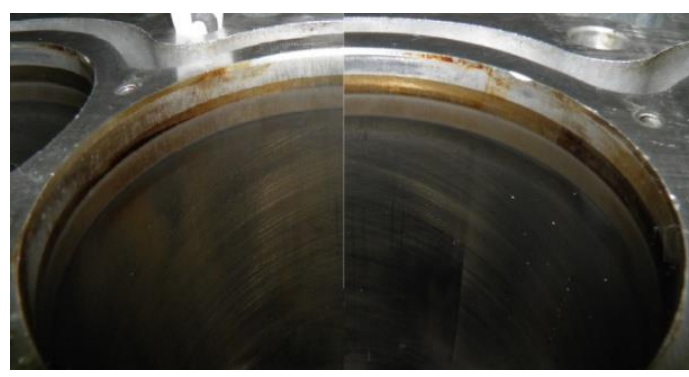

(a)

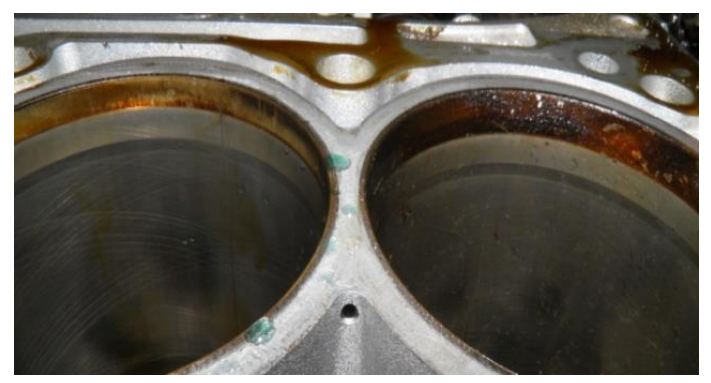

(b)

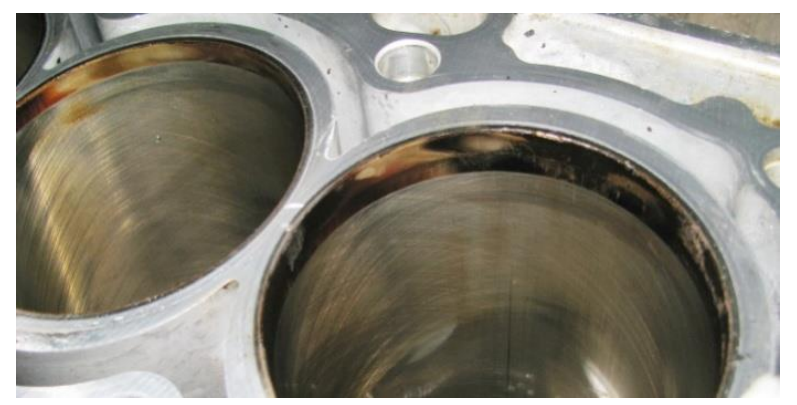

(c)

Figure 2. The expansion of the carbon zone in the upper part of the cylinder caused by its operating with the connecting rod having been deformed as a result of hydrolock $[3,8,9]$.

When the deformed connecting rod is shortened by more than 3-4 $\mathrm{mm}$, the engine will no longer be functional, and the carbon zone in the upper part of the cylinder will not expand downwards for obvious reasons. However, in other cases, when the deformation is relatively small, the damage becomes latent - the engine might stay functional, but the fatigue failure of the rod becomes almost inevitable (most often it appears on the stem average cross section, where the stress is at its maximum due abnormal bending).

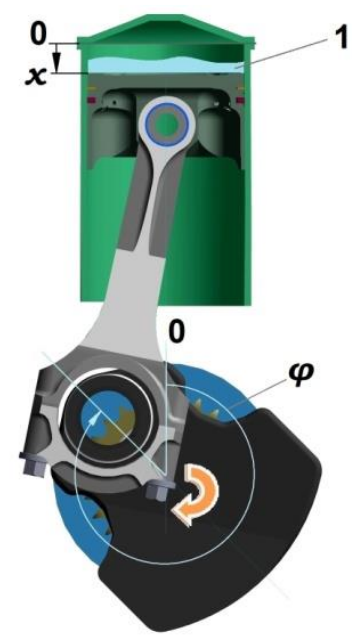

(a)

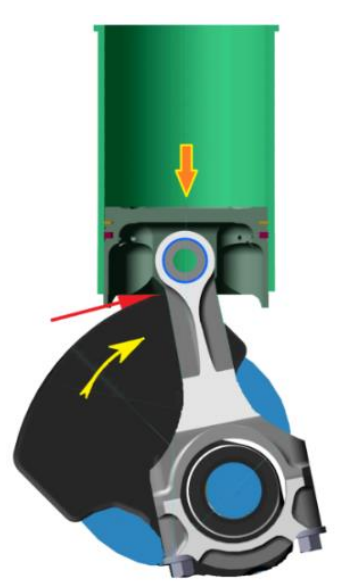

(b)

Figure 3. The general scheme of hydrolock and conrod buckling when incompressible liquid (1) enters into the cylinder (a) and the crankshaft wedging when the connecting rod stem is shortened due to buckling (b).

Considering the features of the known cases of connecting rod deformations caused by hydrolock, it can be assumed that the bending and shortening is proportional to the amount of liquid that has entered the cylinder. However, it remains unclear how much liquid is needed to cause a certain deformation. 


\section{The Calculating Method of Air Parameters during its Compression in a Cylinder with Liquid}

The task of determining the deformation of the connecting rod when the liquid enters the cylinder can be divided into several stages. At the first stage it is necessary to find and consider all the geometric dimensions and kinematic parameters associated with the movement of the piston in the cylinder when there is liquid in it (Figure 3).

This will allow composing the equations for calculation the gas parameters in the cylinder, after which it is possible to get the desired connecting rod deformation.

It should be noted that similar tasks for determining the parameters of air in the cylinder have long been solved, and on the basis of their solution, a number of standard software applications have been developed for calculating the ICE cycle [10]. However, it is not possible to use them for the considered process of air compression with a given amount of liquid, since they do not take into account the influence of liquid on the compression process.

As a result, the solution of the task of air compression in a cylinder with liquid requires the development of an appropriate computational procedure.

To solve the problem of air compression with the liquid in the cylinder it is necessary to begin by making simplifying assumptions that will make it possible to compose the corresponding equations, but at the same time will not have a noticeable negative impact on the result. So, the liquid trapped in the cylinder is considered incompressible, the physical properties of the liquid do not depend on the temperature and pressure of the air due to the rapidity of the process, the temperature and the properties of the liquid are assumed to be unchangeable and constant in all of its volume, and in the process under study, the liquid does not incur evaporation, condensation, chemical reactions and other types of conversion.

Besides, the ignition and combustion of fuel are ignored (for example, in spark ignition internal combustion engines, any liquid from the above shunts the spark plug gap and prevents spark discharge. In diesel engines, the situation is more complicated, but the liquids in question can either prevent the igniting or make it unstable), air and liquid leakage from the cylinder, including leakages through piston rings and valves are not taken into account, and the instantaneous parameters of air in the cylinder are the same in terms of volume, their change by the crankshaft rotation angle occurs quasi-stationary. In addition, the crankshaft rotation speed is constant and does not depend on the liquid that enters the cylinder; this is equivalent to the assumption that the amount of liquid is very little and/or the crankshaft is heavy and/or its inertia is very high during rotation.

Let us now consider the process of air compression with liquid in the cylinder from the moment the intake valves close; first of all, it is necessary to specify the geometrical parameters. The current air volume in the cylinder is:

$$
V=x \pi / 4 D^{2}+V_{k c}(1-\varepsilon)
$$

where, $x$ is the current displacement of the piston, measured from the top dead center, $D$ is the diameter of the cylinder, $V_{k c}$ is the volume of the combustion chamber, $\varepsilon=V_{v} / V_{k c}$ is the relative volume of the liquid (relative to the chamber volume), or the fill factor of the combustion chamber with a liquid.

Considering the fact that the geometric compression ratio is:

$$
r_{c}=V_{h} / V_{k c}+1
$$

where, $V_{h}=S \pi D^{2} / 4$ is the working volume of the cylinder, $S$ is the piston stroke, we can get the value of the current volume of air in the cylinder in the following form:

$$
V=V_{h}\left(x / S+\frac{1-\varepsilon}{r_{c}-1}\right)=V_{h} A_{\varphi}
$$

where $A_{\varphi}=\left(x / S+\frac{1-\varepsilon}{r_{c}-1}\right)$. From the Eq. (2) it is seen that an increase in the amount of liquid leads to a decrease in the volume of air in the cylinder due to it being replacement by fluid.

The relative piston bottom position, which is included in the Eq. (2), is found by the Equations describing the kinematics of the crank mechanism of the internal combustion engine [11], depending on the crankshaft rotation angle $\varphi$, measured relatively to the top dead center:

$$
x / S=0,5\left[(1-\cos \varphi)+\lambda_{c} / 4(1-\cos 2 \varphi)\right]
$$

where $\lambda_{c}=r / L_{c}$ is the relative elongation of the connecting rod with length $L_{c}, r$ is crank radius $(r=S / 2)$. Then the coefficient $A_{\varphi}$, included in the Eq. (2) can be obtained with help of the Eq. (3) in the form of Eq. (4) as: 


$$
A_{\varphi}=0,5\left[(1-\cos \varphi)+\lambda_{c} / 4(1-\cos 2 \varphi)\right]-(1-\varepsilon) /\left(r_{c}-1\right)
$$

Let us now consider the air in the cylinder. According to the first law of thermodynamics as applied to the considered compression process in a cylinder [12], the change of the air's internal energy $d U$ over the process time $d \tau$ as a result of the work $d W$ and the heat extraction $Q_{w}$ can be represented as:

$$
d U=d W-Q_{w} d \tau
$$

Equation (5) can be rewritten in the following form:

$$
m C_{p} d T=-p d V-\alpha_{w} F_{w}\left(T-T_{w}\right) d \tau
$$

where, $d W=-p d V$ is the thermodynamic work, $Q_{w}=\alpha_{w} F_{w}\left(T-T_{w}\right)$ is the amount of heat taken from air to the walls, $\alpha_{w}$ is the heat transfer coefficient, $F_{w}=\pi D(0,5 D+x)$ is the area of the cylinder walls, $d U=m C_{p} d T$ is the change of internal energy in the considered process, $C_{p}$ is the heat capacity of air, $m$ is the air mass in cylinder (which does not change during compression, since there are no leaks from the cylinder).

The equation for $d V$ can be obtained from Eqs. (2) and (4):

$$
d V=V_{h} d A_{\varphi}=V_{h} B_{\varphi} d \varphi
$$

where, the coefficient $B_{\varphi}$ equals:

$$
B_{\varphi}=0,5\left(\sin \varphi+\lambda_{c} \sin 2 \varphi\right)
$$

Thus, taking into account the fact that $\varphi=\omega \tau$, where $\omega=\pi n / 30$ is the angular crankshaft velocity, $n$ is the rotational speed, rpm, the Eq. (6) considering the Eq. (7) can be rewritten as:

$$
\frac{d T}{d \varphi}=-T \frac{R B_{\varphi}}{C_{p} A_{\varphi}}-\frac{30 \alpha_{w} F_{w}}{\pi n m C_{p}}\left(T-T_{w}\right)
$$

Let us now write the equation of state that relates the pressure $p$, temperature $T$ and volume $V$ of air in the cylinder:

$$
p V=m R T
$$

where, $R$ is the gas constant of air. Differentiation of the Eq. (10) gives:

$$
p d V+V d p=m R d T
$$

whereas,

$$
d p=m R d T /{ }_{V}-p^{d V} / V
$$

or

$$
\frac{d p}{d \varphi}=m \frac{R}{V} \frac{d T}{d \varphi}-\frac{R}{V} \frac{d V}{d \varphi}
$$

Substituting expression (9) for $d T / d \varphi$, we obtain:

$$
\frac{d p}{d \varphi}=-p \frac{B_{\varphi}}{A_{\varphi}}\left(1+\frac{R}{C_{p}}\right)-\frac{30 \alpha_{w} F_{w} R}{\pi n m C_{p} A_{\varphi}}\left(T-T_{w}\right)
$$


Let us now simplify the system of Eqs. (9) and (12), bringing them to the following form:

$$
\begin{gathered}
d T / d \varphi=-T \gamma \psi, \\
d p / d \varphi=-p \gamma\left(\psi+\frac{C_{p}}{R}\right)
\end{gathered}
$$

where the coefficients are:

$$
\psi=1+\frac{30 \alpha_{w} F_{w} R}{\pi n p V_{h} B_{\varphi}}\left(T-T_{w}\right), \quad \gamma=\frac{R B_{\varphi}}{C_{p} A_{\varphi}}
$$

and the coefficients $A_{\varphi}$ and $B_{\varphi}$ are found by Eqs. (4) and (8), respectively. To determine the heat transfer coefficient from the air into the walls, Woschni's Equation $[12,13]$ is used as in Eq. (14):

$$
\alpha_{w}=130 \frac{\left(p 10^{-5}\right)^{0,8} \omega_{m}^{0,8}}{T^{0,53} D^{0,2}}
$$

where for the compression stroke $\omega_{m}=2,28 C_{m}$, the average piston speed is $C_{m}=S n / 30$.

The system of Eq. (13) can be solved numerically with initial conditions; the solution is presented in the numerical values of pressure and temperature in the function of the crankshaft rotation angle:

$$
p, T(\varphi)=\int f(p, T, \varphi, \ldots) d \varphi
$$

In the first approximation, this can be done by numerical integration of Eq. (13) using the simple Euler method. To do this, it is needed to set the integration step with respect to the crankshaft rotation angle $\Delta \varphi$, the initial values of temperature and air pressure in the cylinder, which correspond to the closing moment of the intake valves, then to calculate the derivatives $d p / d \varphi$ and $d T / d \varphi$, and after that to determine the pressure and temperature in the next step:

$$
p=p+d p / d \varphi \Delta \varphi, \quad T=T+d T / d \varphi \Delta \varphi, \quad \varphi=\varphi+\Delta \varphi
$$

Initial conditions can be set when the values of the parameters in the steady-state cycle of the internal combustion engine, preceding the hydrolock, are known. However, in order to do this, it is necessary to determine the effect of liquid on the inlet process. If we consider in detail the entire process of the liquid entering the cylinder during the intake stroke, the task will be considerably more complicated. But since we are talking about an approximate model, we can make a simplifying assumption that, for example, the volume of liquid is small compared to the volume of the cylinder (which is quite acceptable with an error that is less than the ratio of the volume of the combustion chamber to the volume of the cylinder, that is, less than $10 \%$ ).

In this case, the liquid entering the cylinder during the intake stroke will not affect the temperature and air pressure in the cylinder during that period. Thus, accepting this condition means in fact that during the intake there was no liquid in the cylinder and it appeared right at the end of the intake, simply replacing a part of the air in the cylinder.

This means, the initial values of pressure and temperature at the moment of the intake valves closing can be easily obtained using well-known standard software for calculating the working cycle of the engine.

\section{Determining the Initial Conditions}

To calculate the initial values of the parameters needed to simulate a hydrolock, the Lotus Engine Simulation software was used $[10,14]$.

The main function of the software is to calculate the instantaneous parameters of the thermodynamic cycle of the internal combustion engine (the temperature and the pressure in the cylinder are average in terms of volume) with respect to the crankshaft rotation angle. In this case, the gas flow in the inlet and exhaust pipes adjacent to the cylinder is calculated as one-dimensional, which makes it possible to explore the dynamic phenomena in the pipelines and their influence on the parameters of air and gas in the cylinder. 


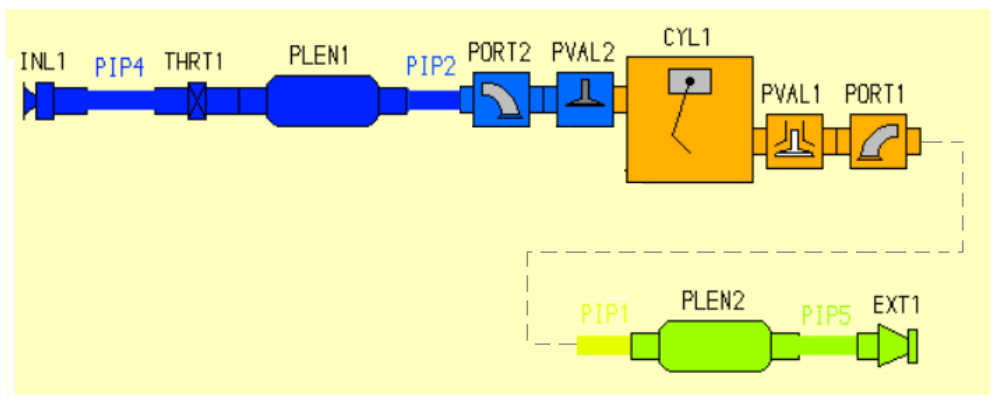

Figure 4. 1-cylinder geometric engine model in the Lotus Engine Simulation software.

Since the purpose was not to calculate the main integral parameters of the engine (power, torque, or specific fuel consumption), but the temperature and pressure in the cylinder, a simplified 1-cylinder model was used (Figure 4).

In order to perform the calculation, a number of parameters were set: the type of internal combustion engine was set to gasoline with the spark ignition, engine dimensions were set to $83 \times 80 \mathrm{~mm}$, compression ratio - to 9.0 , operating mode - to 3,000 rpm (the calculation was made according to the standard software procedure for the speed rotation range of 1,000-6,000 rpm, with the step of $1000 \mathrm{rpm})$, the closing of the intake valves - to $40^{\circ}$ after the bottom dead center $\left(220^{\circ}\right.$ from the top dead center), the walls temperature - to $390 \mathrm{~K}$ as well as some others.

The calculation of the cycle was carried out taking into account the heat exchange with the walls (the software considers the heat exchange of gas with the walls of the combustion chamber and the piston as well as the heat loss to the cooling fluid).

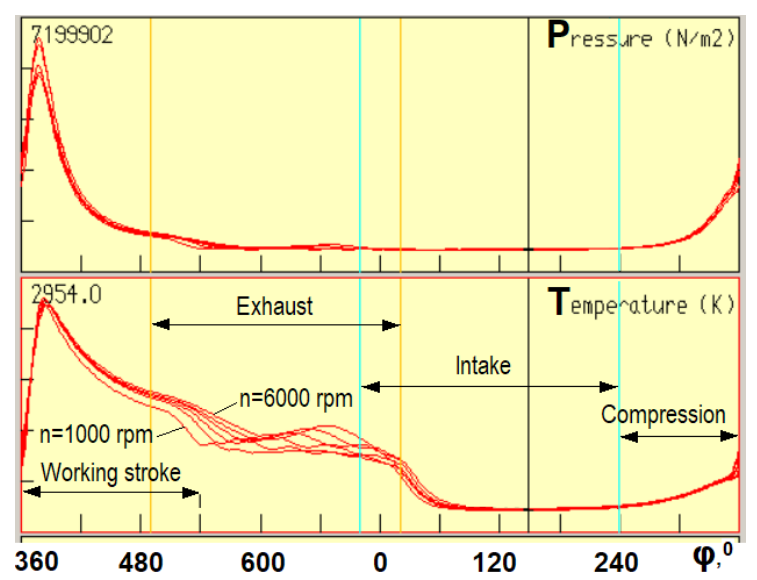

Figure 5. Diagrams of pressure $\boldsymbol{p}$ and temperature $\boldsymbol{T}$ in the cylinder of the engine by the crankshaft rotation angle $\boldsymbol{\varphi}$ at 1,000-6,000 rpm, obtained using the Lotus Engine Simulation software.

Figure 5 presents the calculated pressure and temperature diagrams in the cylinder at modes ranging from 1,000 to $6,000 \mathrm{rpm}$, including the selected mode of 3,000 rpm. These data made it possible to establish the initial pressure and temperature values corresponding to the closing moment of the inlet valves, which were necessary for subsequent modeling of the hydrolock by the means of calculating the compression stroke in the presence of liquid (for the provided example $p_{0}=1,23$ bar,$T_{0}=363 \mathrm{~K}$ at $\varphi=220^{\circ}$ were obtained).

It is characteristic that this method of selecting the initial values of pressure and temperature almost completely corresponds to the actual operating conditions of the internal combustion engine when hydrolock occurs; the cycle preceding the ingress of liquid into the cylinder is no different from normal and in fact determines the initial conditions for hydrolock (given the amount of liquid is small).

\section{Model Error Analysis}

Figure 6 shows the result of calculating the pressure and temperature in the cylinder during the compression stroke in the absence of liquid according to the developed method for the step with respect to the angle $\varphi=2^{0}$. The diagrams of pressure and temperature, which were calculated by the Lotus Engine Simulation software and accepted as etalon, are placed here too. The comparison was made in order to estimate the possible error in the calculation of the compression process, since the program gives the calculation of a complete closed loop, and the suggested method is only a part of it.

Indeed, for cyclic calculations, the parameters at the output of each cycle are the initial conditions for the next cycle, which, after a certain number of cycles, eliminates the influence of the initial conditions. On the contrary, only a part of 
the full cycle is implemented in the suggested method. And as it is known, in an open cycle, you can expect an increase in the error, especially when using simple methods of numerical integration, which can make the results of the calculating points that are far from the initial conditions unreliable.

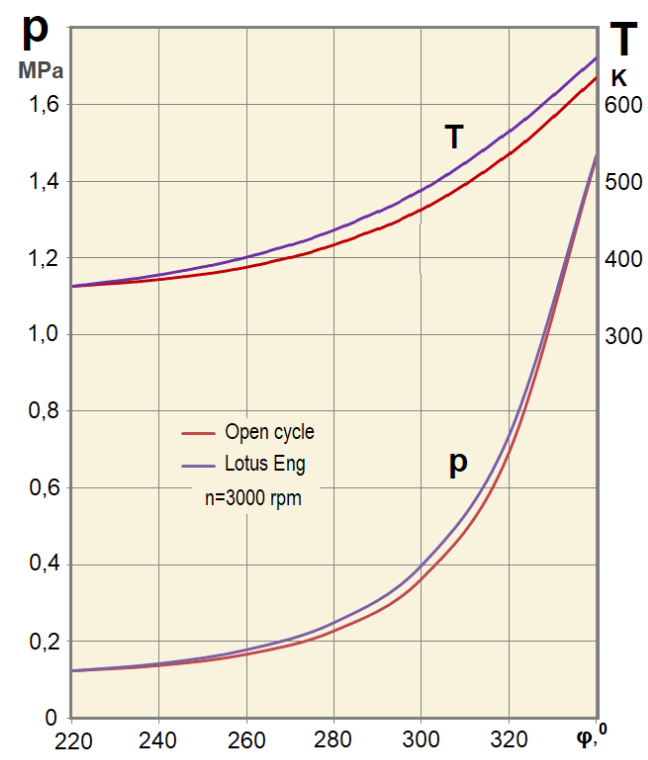

Figure 6. Comparison of the calculation results of the compression process in the cylinder without liquid using the developed method (open cycle) and the Lotus Engine Simulation software.

Nevertheless, the developed method gave quite satisfactory results. For example, the pressure error, i.e. the difference between the values given by the suggested method and the software does not exceed $-8.5 \%$ (the error gives smaller value), and when getting closer to the top dead center the error in calculating the pressure decreases to $-3.7 \%$. The temperature is calculated more precisely: with the same error of $-3.7 \%$ near the top dead center, the error in the calculation by the method in the middle phase of the compression process does not exceed $-5 \%$. This result gives grounds to apply the developed method to simulate the process of compression with liquid.

\section{Connection between Pressure in the Cylinder and Buckling of the Conrod Stem}

As shown above (Figure 1), the damage to the internal combustion engine when liquid enters the cylinder occurs mainly due to the buckling of the connecting rod stem. It is clear that sufficiently accurate solutions to this problem can be obtained using the finite element model (FEM) [4, 15], however, within the framework of the assumptions made above, it is possible to find approximate and simpler, but quite reliable solutions for practical application.

If, for the compression process in the cylinder, the inertia forces affecting the connecting rod are neglected (which is quite true if the fill factor is noticeably higher than zero and the crankshaft rotation speed is small), then the axial compression force affecting the conrod stem is determined by the pressure in the cylinder $p$ and equals:

$$
R=\left(p-p_{0}\right) F
$$

where $F=\pi D^{2} / 4$ is the piston area, $p_{0}$ is the pressure in the crankcase (in the first approximation, it can be taken equal to the ambient pressure).

The compression force is perceived by the cross section area of the stem (Figure 7):

$$
A=\left(h-h_{1}\right) b-h_{1} t
$$




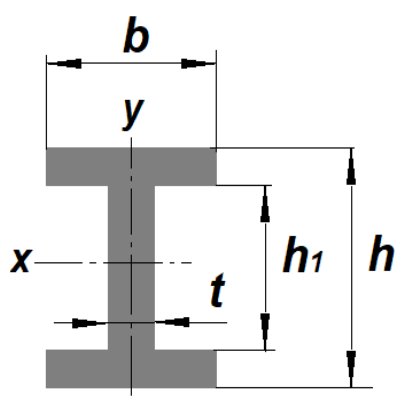

Figure 7. Conrod stem cross section: $\boldsymbol{h}$ is the profile height, $\boldsymbol{b}$ is the profile width and $\boldsymbol{t}$ is the profile thickness.

Then, obviously, the compressive stress in the stem is:

$$
\sigma=R / A=\left(p-p_{0}\right)^{F} / A
$$

The compression force and the stress in the stem can easily be obtained from the calculation results of the pressure in the cylinder. On the other hand, as follows from the theory [16], with an increase in the axial load on the rod, when the compression stress $\sigma$ in the stem material exceeds a certain critical value $\sigma_{c r}$, buckling occurs.

The critical stress value $\sigma_{c r}$ depends on the flexibility of the stem [17]:

$$
\lambda=\mu l / i
$$

where, $l$ is the length of the stem, $i$ is the inertia radius of the stem cross section, and $\mu$ is the length reduction factor [16]. The conrod stem inertia radius is:

$$
i=\sqrt{I / A}
$$

where the moments of inertia [18] along $\boldsymbol{x}$ and $\boldsymbol{y}$ axes (Figure 11) are:

$$
I_{x}=0.0833\left[b h^{3}-(b-t) h_{1}^{3}\right], I_{y}=0,0833\left[\left(h-h_{1}\right) b^{3}+h_{1} t^{3}\right]
$$

In case of the strain in the direction along the axis of the crankshaft, the conrod stem at the edges can be considered clamped, if we neglect the end-plays of the conrod-piston group parts; in this case: $\mu_{y}=1 / 2$. In the perpendicular direction, the conrod stem has a hinged attachment in the swing plane (the conrod has the ability to rotate freely in the crank and piston heads), in this case $\mu_{x}=1$.

Once the dimensions of the stem are known, it is easy to calculate its flexibility. As an example, we can consider a conrod stem with the dimensions typical for gasoline internal combustion engines: $l=100 \mathrm{~mm}, h=20 \mathrm{~mm}, h_{1}=$ $14 \mathrm{~mm}, b=12 \mathrm{~mm}, t=4 \mathrm{~mm}$. Then, according to the Eq. (19) the values of flexibility are: $\lambda_{x}=12,96 ; \lambda_{y}=16,62$, whence it follows that with given dimensions, the flexibility in the direction of the crankshaft axis is greater, and it is in this direction that one should expect the stem strain with buckling (which corresponds to the type of conrod deformation shown in Figure 1). By reducing the height of the stem profile $h$ the flexibility $\lambda_{x}$ will increase, and buckling may occur in the cross direction - in the swing plane of the connecting rod (Figure 2).

Within the range of the obtained values of flexibility, the critical stress is close to the yield strength of the material, but somewhat less. According to the classic theory [16], it is possible to consider the influence of the stem flexibility on the critical stress by the means of the permissible stress reduction factor $\varphi_{\sigma}$, i.e.:

$$
\sigma_{c r}=\sigma_{t} \varphi_{\sigma}
$$

where $\sigma_{t}$ is the yield strength of the connecting rod material. It is known that chromium-nickel steels are often used for ICE conrods of this type. At the above-mentioned values of flexibility $\varphi_{\sigma}=0,96$ and with the heat treatment up to the hardness of HB 280-320, the yield strength for such steels will be approximately $800 \mathrm{MPa}$ [19]. This follows thence that the critical compressive stress for the conrod made of such steel is $\sigma_{c r}=770 \mathrm{MPa}$. 


\section{RESULTS AND DISCUSSION}

The calculation of the air compression process in the cylinder with the above-mentioned initial conditions within the range of crankshaft rotation angles from the moment of the intake valves closing $\left(\varphi=220^{\circ}\right)$ to the top dead center was performed for the various values of the fill factor of the combustion chamber with liquid $\varepsilon$.

The accepted values of $\varepsilon=0-1,2$ enabled us to obtain a pressure change in the cylinder (Figure 10) in a wide range of states, from the clean air being compressed without liquid to the air being compressed with liquid exceeding the volume of the combustion chamber by $20 \%$.

The calculation results (Figure 8) show that the pressure in the cylinder begins to increase markedly compared to conventional compression without liquid only $50-60^{\circ}$ before the top dead center; at $10^{\circ}$ before the top dead center when the combustion chamber is filled with liquid at a considerable level, the pressure may increase ten- or even several hundred-fold.

It is clear that such significant increase in pressure that occurs due to the presence of a large amount of liquid in the cylinder can cause damage to parts, however, having the data on the pressure alone cannot be considered sufficient. In order to determine the limit value of the fill factor at which the engine begins to take damage it is necessary to relate the calculation results to the durability of the engine parts.

Figure 9. is represented by a diagram of stresses in the conrod stem, calculated by the Eq. (18). The diagram shows the value of the critical stress $\sigma_{c r}$, above which the area of the stem buckling is located.

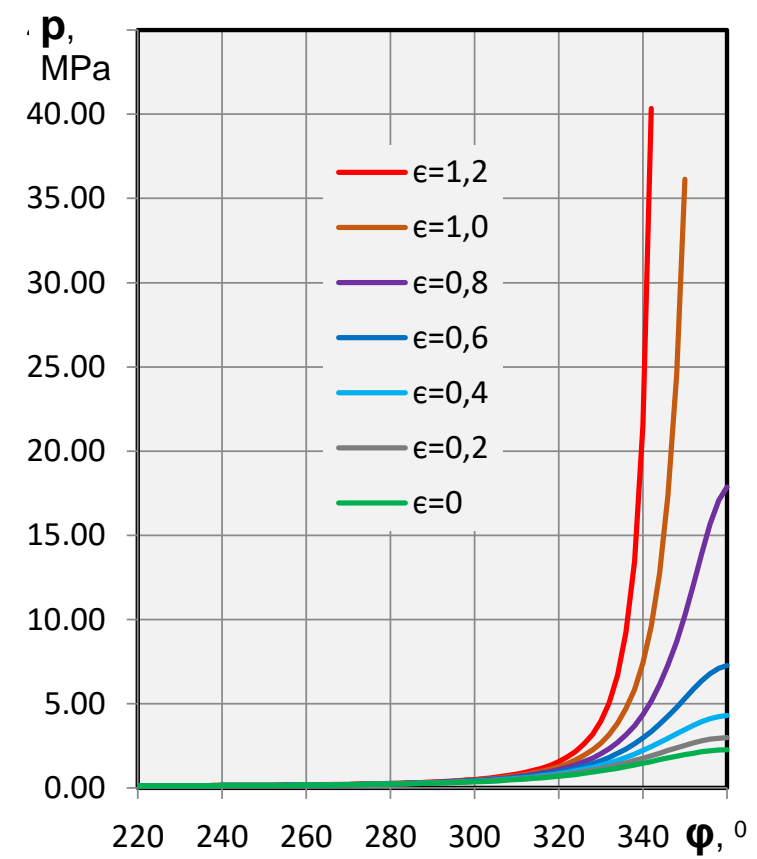

Figure 8. The change in the cylinder pressure with respect to the crankshaft rotation angle with the piston being near the top dead center $\left(360^{\circ}\right)$, at the different values of the liquid fill factor $\boldsymbol{\varepsilon}$ of the chamber.

The diagram also shows that the intersection of the stress curves with the critical voltage line strictly corresponds to a certain crankshaft rotation angle and the piston position. It can be assumed that when the piston reaches this coordinate during compression, the connecting rod actually loses its strength - the piston remains in place, but the crankshaft continues to rotate, while the connecting rod keeps on getting plastically deformed (shortened) until the conrod journal of the crankshaft passes the position that matches the top dead center. 


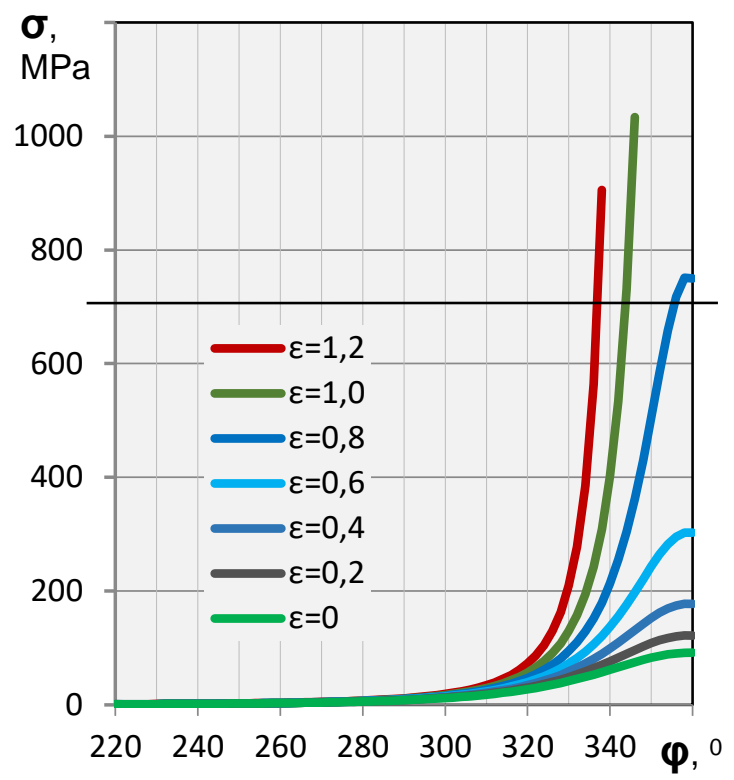

Figure 9. The change in the compression stresses in the conrod stem with respect to the crankshaft rotation angle with the piston being near the top dead center $\left(360^{\circ}\right)$, in its relation to the fill factor of the combustion chamber.

It is clear that elasticity can lead to a slight decrease in residual deformation after the removal of the load. However, with significant deformation (Figure 1) in the 1st approximation, in case of the described process of plastic deformation of the conrod stem it appears fair to neglect the elasticity and consider the deformation after buckling only as plastic [16].

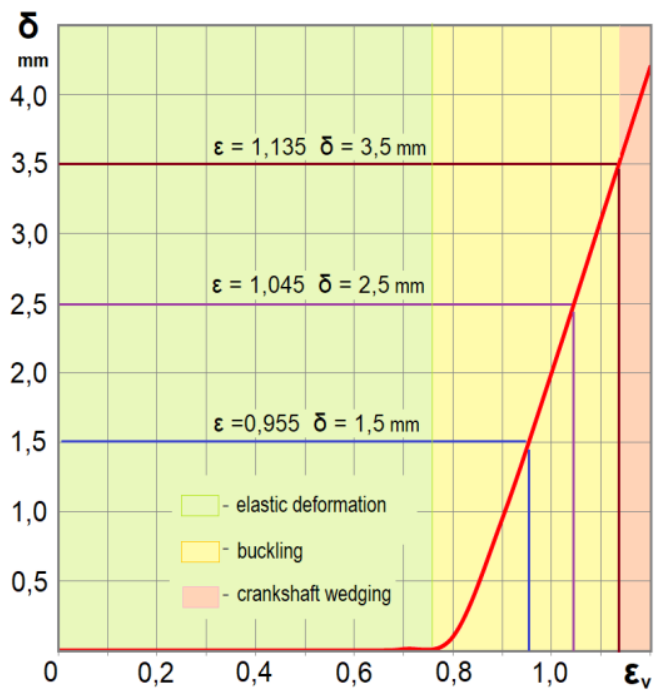

Figure 10. The value of the longitudinal deformation (shortening) of the connecting rod that occurs as the result of hydrolock in the ICE cylinder can be divided into three zones by the liquid filling ratio.

Then, according to the calculation results (Figure 9), when buckling occurs, the value of longitudinal deformation is easy to calculate - it will be equal to the coordinate of the piston or, equivalently, to the distance from the piston to the top dead center at the moment of buckling of the conrod stem.

The results of calculating the longitudinal deformation of the connecting rod stem in dependance to the filling of the combustion chamber with liquid are shown in Figure 10. The data obtained showed that the hydrolock in the cylinder of the internal combustion engine actually occurs under the condition that the combustion chamber is almost completely filled with liquid, and the onset of plastic deformation is observed at $80 \%$ filling (this can also be seen in Figure 9).

With the chamber being filled up to the range of $80-110 \%$, the engine might remain functional after hydrolock has occurred, even in spite of the connecting rod longitudinal deformation of up to $3.5 \mathrm{~mm}$, however, that will shortly lead to the destruction of the connecting rod stem from abnormal bending loads [9, 20]. Meanwhile, in case the chamber is filled with liquid to more than 110-120\% of its volume, an extremely large deformation will follow and the engine will most likely fail immediately after hydrolock has occurred due to the piston wedging the crankshaft in the bottom dead center. 
To summarize, the quantitative data on the conrod deformation at the time of hydrolock, on the one hand, match the experimental data obtained in the studies of failure causes in real engines [8], and on the other hand, make it possible to apply the hydrolock quantitative characteristics, which have not existed to date. This is of practical importance in the event of problems related to determining the engine failure causes. In addition, the results of the calculation of the conrod deformation enable us to relate the moment of hydrolock and the amount of liquid that caused it to damage consequences, including the characteristics of the fatigue failure of the deformed conrod under the influence of abnormal bending loads (if the damaged engine remains operational and continues to operate).

\section{CONCLUSION}

The present study provides a thorough analysis of the features of conrod damage caused by hydrolock as well as develops a method for calculating the conrod stem deformation when liquid enters the cylinder ICE (hydrolock) and presents the first quantitative data on the dependence of the stem deformation from the liquid filling coefficient of the combustion chamber. The calculations have demonstrated that the practically observed deformation of the connecting rod with the buckling of the stem occurs when the combustion chamber is filled with liquid to a minimum of $80 \%$. With the further increase in the amount of liquid in the range of up to $110 \%$ of the combustion chamber volume, the connecting rod deformation increases, but normally does not interfere with the performance of the internal combustion engine. However, when the relative liquid filling of the chamber is over 110-120\%, the deformation of the connecting rod reaches such values that after hydrolock occurs the engine may break down due to the piston wedging the crankshaft at the bottom dead center.

There is a scope for further study of the nature of the hydrolock-related deformation in such parts as the piston, the piston pin and the cylinder using stress-strain simulation software based on the finite elements method, which seems to be the most effective way to investigate the failure causes in various types of internal combustion engines.

\section{REFERENCES}

[1] E. Greuter and S. Zima, Engine Failure Analysis. Internal Combustion Engine Failure and Their Cause. Warrendale, USA: SAE International, 2012.

[2] Piston damage - recognizing and rectifying. Service tips \& infos, Article No. 50003 973-02, Neuenstadt, Germany: MS Motorservice International GmbH, 2016.

[3] A. E. Khrulev, "Methods of study and determination of the causes of heavy duty damages of internal combustion engines," Theses of the Int. Sci. and Tech. Conf. "Engine-2017", Moscow State Technical University (MSTU) N.E.Bauman, 2017, pp. $22-23$.

[4] P. S. Kumar and K. Kumar, "Buckling Analysis and Shape Optimization of Connecting Rod using FEA," REST J. on Emerg. trends in Model. and Manuf., vol. 2, no. 2, 2016, pp. 44-50.

[5] P. S. Shenoy and A. Fatemi, "Dynamic analysis of loads and stresses in connecting rods," Proceedings of the Institution of Mechanical Engineers, J. Mech. Eng. Sci., vol. 220, no. C, 2006, pp. 615-624, doi: 10.1243/ 09544062JMES105.

[6] M. umar and S. N. Prajapati, "Design, Buckling and Fatigue Failure Analysis of Connecting Rod: A Review," Int. J. Adv. Eng. Res. Sci., vol. 4 no. 7, 2017, pp. 39-44, doi: 10.22161/ijaers.4.7.7.

[7] K. L. Nagaraju and R. Chandan, "Buckling Analysis Of Connecting Rod," Int. Res. J. Eng. Tech., vol. 03, no. 08, 2016, pp. 1358-1361.

[8] A. Khrulev, S. Losavio, V. Drozdovsky, Expertise of technical condition and fault causes of automotive technics, Moscow, Russia: Publishing house ABS, 2019.

[9] A. Khrulev and S. Samokhin, "Hydrolock delayed action," Automobile and Service, no. 08, 2011, pp. 36-39.

[10] L. V. Grekhov et al., Mechanical Engineering. Internal combustion engines, Vol. IV - 14. Moscow, Russia: Mashinostroenie, 2013.

[11] J. B. Heywood, Internal Combustion Engine Fundamentals, Series in Mechanical Engineering. New York, USA: McGrawHill Inc., 1988.

[12] V. G. Dyachenko, Theory of internal combustion engines. Kharkov, Ukraine: HNADU, 2009.

[13] A. M. Levterov and L. I. Levterova, "Analysis of mathematical models of the mechanism of soot formation during the combustion of hydrocarbon fuels," News of National Technical University "KhPI". Series: Mathematical Modeling in tech and technology, vol. 5 no. 979, 2013, pp. 130-141.

[14] B. Duleba, "Simulation of Automotive Engine in Lotus Simulation Tools," Transfer inovacii, vol. 30, 2014, pp. 48-52.

[15] M. Moatamedi and H. Khawaja, Finite Element Analysis. New York, USA: CRC Press, 2018.

[16] V. I. Feodosyev, Resistant materials. Moscow, Russia: MSTU N. Bauman, 2018.

[17] J. M. Gere, Mechanics of Materials, 6th ed. Belmont, USA: Brooks/Cole -Thomson Learning, 2004.

[18] M. V. Favorin, Moments of bodies inertia. Moscow, Russia: Mashinostroenie, 1977.

[19] V. G. Sorokin and M. A. Gervas'ev, Steels and alloys. Moscow, Russia: Intermet Engineering; 2001. 
[20] A. E. Khrulev and Y. V. Kochurenko, "Method for determining the cause of the ICE failure for severe damages in operation," Int. Comb. Eng., vol. 1, 2017, pp. 52-60, doi: 10.20998/0419-8719.2017.1.10. 